\title{
Frequency Sweep Linearization for Semiconductor Laser Using a Feedback Loop Based on Amplitude-Frequency Response
}

\author{
Jianfang Zhu, Ligong Chen \\ Liangjiang International College, Chongqing University of Technology, Chongqing, China \\ Email: zjf859590119@163.com, chenligong@cqut.cn
}

How to cite this paper: Zhu, J.F. and Chen, L.G. (2021) Frequency Sweep Linearization for Semiconductor Laser Using a Feedback Loop Based on Amplitude-Frequency Response. Optics and Photonics Journal, 11, 273-283.

https://doi.org/10.4236/opj.2021.118017

Received: November 6, 2020

Accepted: July 31, 2021

Published: August 3, 2021

\begin{abstract}
A scheme of frequency sweep linearization of semiconductor lasers using a feed-back loop based on amplitude-frequency response is demonstrated in this paper. The beat frequency signal is obtained by self-heterodyne detection. The frequency changes are converted to the envelope of beat frequency signal after amplitude-frequency response. The active frequency sweep linearization is realized by feeding envelope deviations back to the drive currents of the lasers by a feedback loop. A simulation model is built to verify this scheme by Simulink. This scheme does not need high-performance, expensive lasers, complex linearization or tedious post-processing processes, which are of great significance for related applications.
\end{abstract}

\section{Keywords}

Frequency Sweep Linearization, Frequency-Modulated-Continuous-Wave, Feedback, Semiconductor Laser

\section{Introduction}

Light detection and ranging (LiDAR) is a kind of radar working in optical band. According to the working waveform, LiDAR can be divided into pulse LiDAR and frequency-modulated-continuous-wave (FMCW) LiDAR. Compared with pulse LiDAR, FMCW LiDAR has many advantages, such as lower power, high resolution, high sensitivity and immune background light. A light source with a linear change in frequency is needed for an FMCW LiDAR. In detection, the reflected light with different flight time is combined with the local reference light on a photodetector to produce beat frequency signal. The beat frequency can be extracted using a Fourier Transform (FT) [1] [2]. Because the beat frequency is 
related to the flight time (or the ranging), so the flight time (or the ranging) is obtained from beat signals. The linearity and bandwidth of frequency sweep for light sources are key parameters for high-precision ranging. However, how to get a low-cost high frequency sweep linearity laser source is still a problem to be solved. To realize frequency sweep in semiconductor laser has flexible tuning characteristic and can be directly tuned by injecting current. Besides, the scheme has the advantages of simple structure, low price, and easily realized. Therefore, the technology of laser frequency sweep linearization of current tuning in semiconductor lasers has attracted much attention. Researchers have proposed to characterize the nonlinearity of frequency modulation with self-heterodyne detection and realize the linearization of slow frequency modulation by an optoelectronic feedback loop [3]. Based on this scheme, an optoelectronic phase-locked loop (PLL) was also added to optoelectronic feedback loop to realize frequency stabilization [4]. Wideband linear frequency sweep based on optoelectronic PLL was also realized and the seep speed can be achieved to $100 \mathrm{GHz} / \mathrm{ms}$ [5]. The frequency sweep linearization based on the phase-locked loop technology mentioned above has high precision, but it also has high requirement for laser line width and complex circuit. Moreover, a frequency discriminating photoelectronic negative feedback system for active control of frequency modulation is also put forward [6]. Although the structure of frequency discrimination photoelectric negative feedback technique is simple, the frequency excursion and modulation period are limited by the relevant devices in the frequency discrimination circuit.

In this paper, we propose a new frequency discrimination scheme, the frequency variation of beat frequency signal is converted into amplitude variation, and the envelope signal is used to represent the frequency modulation nonlinearity. The deviation signal is regarded as a voltage signal (error signal) to change the drive current. Simulation results show that the frequency modulation linearization is carried out effectively in this scheme.

\section{Influences of Frequency Sweep Linearization in FMCW LiDAR}

A schematic diagram of a triangular FMCW LiDAR is shown in Figure 1. Probe light from the target interferes with the reference and the beat signal is recorded by a detector [7].

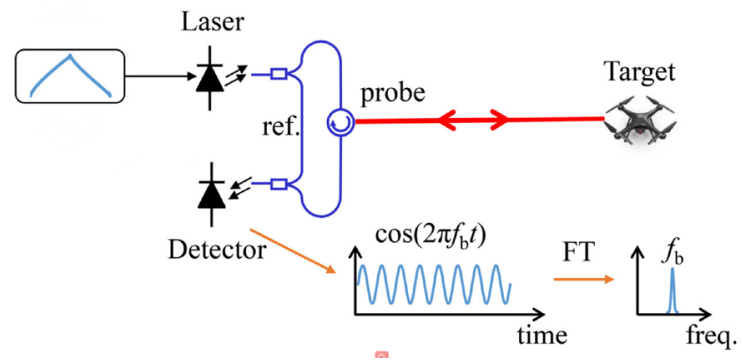

Figure 1. Schematic diagram of FMCW LiDAR. 
Since the laser frequency sweep is linear, the beat signal frequency $f_{b}$ is proportional to the target distance $D$, is given by

$$
D=\mathrm{c} \times \tau / 2=\mathrm{c} \times f_{b} /(2 \gamma),
$$

where $\mathrm{c}$ is the speed of light, $\gamma$ is the laser frequency sweep rate and $\tau$ is the relative delay time between the transmitter and receiver, $\tau=2 D / c=c \times f_{b} / \gamma$. The distance detection resolution $\Delta D$ is given by

$$
\Delta D=\mathrm{c} \times \Delta f_{b} /(2 \gamma),
$$

where $\Delta f_{b}$ is the spectral width of the beat signal.

Figure 2 is the ranging principle of triangular FMCW LiDAR. In the case of ideal triangular wave linear frequency sweep, the bandwidth of the beat signal is narrow, as shown in Figure 2(a). While the nonlinearity of frequency sweep leads to the broadening of spectral width which is shown in Figure 2(b) [8].

In the ideal case, the output frequency of distributed feedback (DFB) laser in the half ramp of up frequency sweep can be expressed as follows

$$
f(t)=f_{0}+2 \mathrm{~B} t / \mathrm{T}, 0 \leq t \leq \mathrm{T} / 2,
$$

where $\mathrm{B}$ is the frequency excursion, $\mathrm{T}$ is the modulation period, $f_{0}$ is the initial frequency of laser. However, in practice, considering the nonlinear characteristics of the laser itself, the nonlinear term $f_{\varepsilon}(t)$ introduced into the output frequency of the laser can be expressed as
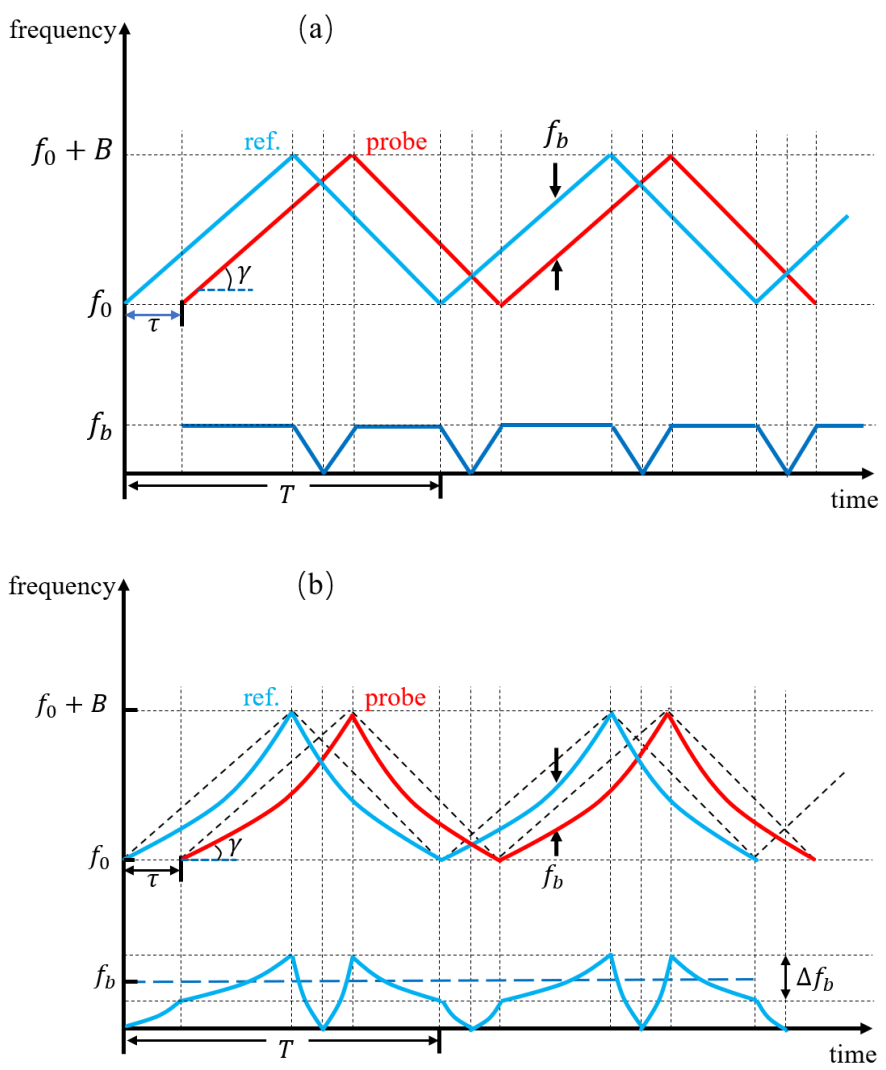

Figure 2. The detection and ranging of a triangular FMCW LiDAR with (a) ideal FMCW and (b) nonlinear FMCW. 


$$
f(t)=f_{0}+2 \mathrm{~B} t / \mathrm{T}+f_{\varepsilon}(t), 0 \leq t \leq \mathrm{T} / 2 .
$$

The output phase of the beat frequency signal can be expressed as follows

$$
\varphi_{b}(t)=2 \pi f_{0} \times \tau+2 \pi \mathrm{B} \times\left(2 t \tau-\tau^{2}\right) / \mathrm{T}+\int_{t-\tau}^{t} 2 \pi f_{\varepsilon}(t) \mathrm{d} t, 0 \leq t \leq \mathrm{T} / 2 .
$$

Because the delay time $\tau$ is very small, the variation of $f_{\varepsilon}(t)$ is very small, as in

$$
\int_{t-\tau}^{t} f_{\varepsilon}(t) \mathrm{d} t=f_{\varepsilon}(t)(t-(t-\tau))=f_{\varepsilon}(t) \times \tau .
$$

The Taylor expansion of Equation (4) is carried out according to $2 t / \mathrm{T}$, ignoring the high-order nonlinearity and retaining only the quadratic nonlinearity, as in

$$
f(t)=f_{0}+\frac{\sum_{i=1}^{2} \mathrm{a}_{i}(2 t / \mathrm{T})^{i}}{\sum_{i=1}^{2} \mathrm{a}_{i}} \times \mathrm{B},
$$

where $\mathrm{a}_{i}{ }^{\prime}=\mathrm{a}_{i} / \sum_{i=1}^{2} \mathrm{a}_{i}$ is the frequency modulation coefficient of Equation (7). The beat frequency can be expressed as

$$
f_{b}(t)=2 \mathrm{~B} \tau / \mathrm{T}+\left(4 \mathrm{a}_{2}{ }^{\prime} t / \mathrm{T}\right) \times 2 \mathrm{~B} \tau / \mathrm{T} .
$$

The first term on the right side of Equation (8) is the ideal beat frequency, and the second term is caused by the quadratic nonlinearity [9]. Therefore, the frequency variation of beat frequency is caused by the nonlinear term. The ranging principle of FMCW LiDAR is based on the continuous linear frequency sweep of laser, and a small nonlinearity will affect the ranging accuracy and distance resolution of FMCW LiDAR.

\section{Model of Frequency Sweep Linearization}

\subsection{Model of Frequency Tuning of DFB Laser}

Under the linear injection current, the output wavelength of an ideal semiconductor laser is continuous linear sweep. But, under the influence of the thermal effect of the injection current and the response hysteresis effect of the laser, the frequency sweep nonlinearity will be very large. According to the relevant research [10], the analytical model of current tuning characteristics of DFB laser should be expressed as follows

$$
\lambda(I, T)=\left(\mathrm{k}_{1} \times T+\mathrm{k}_{2}\right) \times I^{2}+\left(\mathrm{k}_{3} \times T+\mathrm{k}_{4}\right) \times I+\mathrm{k}_{5} \times T+\lambda_{0},
$$

where $T$ is the temperature and the undetermined constants $\mathrm{k}_{1}, \mathrm{k}_{2}, \mathrm{k}_{3}, \mathrm{k}_{4}$, $\mathrm{k}_{5}$ and the initial wavelength $\lambda_{0}$. From the analysis of Equation (4), (7), (9), it can be concluded that the relationship between the injection current $I(t)$ and the output frequency $f(I(t), \mathrm{T})$ is a quadratic function. When $I(t)=\mathrm{I}_{0}+\alpha t$, the output laser frequency is given by

$$
f(I(t), \mathrm{T})=f_{0}+\mathrm{a} \times I(t)+\mathrm{b} \times I^{2}(t)=f_{0}+\mathrm{a} \times \alpha t+\mathrm{b} \times \alpha^{2} t^{2},
$$

where the temperature $\mathrm{T}$ is assumed to be stable, temperature tuning is included in the initial frequency $f_{0}$. The initial current $\mathrm{I}_{0}$ is assumed to be 0 and $\alpha$ is 
the increasing rate of current, the undetermined coefficients $\mathrm{a}$ and $\mathrm{b}$ are related to the frequency modulation coefficients $a_{i}^{\prime}$. In the next simulation scheme, we will assume the relevant parameters and coefficients (Table 1).

\subsection{Scheme of Frequency Sweep Linearization}

Figure 3 shows the schematic diagram for scheme of frequency sweep linearization. The waveform generator generates a standard triangular drive current $I(t)$ to directly modulate the laser. The output beam is sent to a Mach-Zehnder interferometer (MZI) with an arm of relative time delay $\tau$ to the reference one. Then the balanced photodetector records the beat signal from the MZI. The beat signal is fed into a high-pass filter operating in the transition band. The amplitude gain $K\left(f_{b}(t)\right)$ of amplitude-frequency response varies with the beat frequency of the beat signal. The high pass filter with better linearity of amplitude-frequency response in the range of beat frequency variation directly determines the inherent residual nonlinearity of the frequency sweep.

On the extraction scheme of envelope, as the beat signal contains low frequency components, the synchronous envelope detection is not feasible. So we design to extract envelope signal by a field programmable gate array (FPGA), and in the following simulation, we use MATLAB program of envelope extraction to extract the envelope signal.

The envelope signal $K\left(f_{b}(t)\right) A(t)$ is extracted and directly converted into a voltage signal. If the original amplitude $A(t)$ of beat signal is not standard, the envelope signal needs to be normalized. The voltage is compared with an ideal standard voltage. The deviation $U=\left(K\left(f_{b}(t)\right) A(t) / A(t)-1\right) A_{\text {offset }}$ is fed back to the laser driving current after integration and gain amplification, so as to realize active frequency sweep linearization.

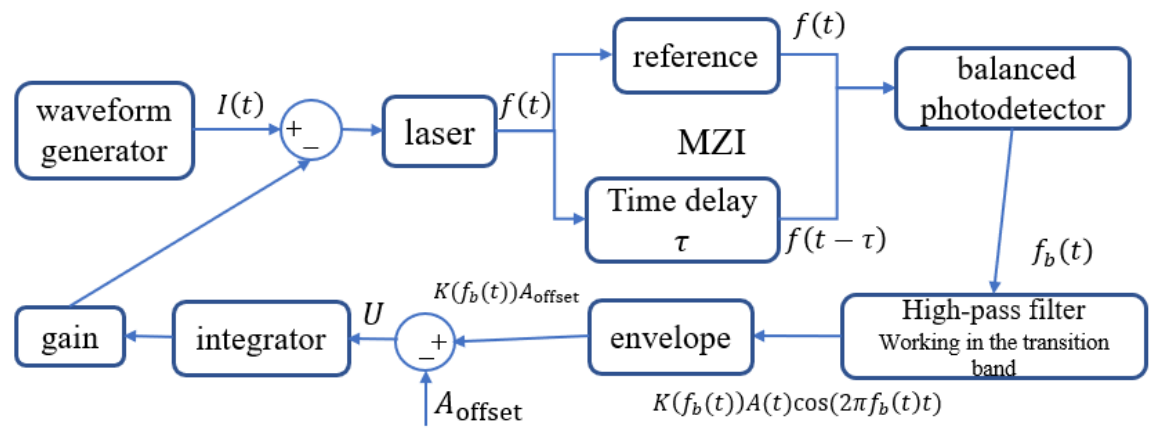

Figure 3. Schematic diagram of frequency sweep linearization.

Table 1. Parameters and values in the model.

\begin{tabular}{ccccc}
\hline parameters & $\mathrm{B}$ & $\mathrm{T}$ & $\tau$ & $f_{0}$ \\
\hline values & $1 \mathrm{GHz}$ & $1 \mathrm{~ms}$ & $2.86 \mu \mathrm{s}$ & $10 \mathrm{GHz}$ \\
parameters & $\mathrm{a}$ & $\mathrm{b}$ & $\mathrm{I}_{0}$ & $\alpha$ \\
values & $0.8 \mathrm{GHz} / \mathrm{mA}$ & $0.2 \mathrm{GHz} / \mathrm{mA}^{2}$ & $0 \mathrm{~mA}$ & $2 \times 10^{3} \mathrm{~mA} / \mathrm{s}$ \\
\hline
\end{tabular}


According to the proposed scheme, we build a simulation feedback system based on tuning characteristics of the semiconductor laser, as shown in Figure 4. The system consists of FMCW electronic signal generation module, delay module, receiving module, feedback module.

Among them, the module of FMCW signal generation is used to generate the standard triangular drive current by waveform generator to modulate laser (FM modulator passband module with the frequency drift coefficient of $1 \mathrm{GHz} / \mathrm{mA}$ with current variation). The delay module is used to simulate the time delay $\tau$ caused by the transmission distance $\mathrm{D}$. The receiving module is used to mix the reflected light (delay signal) with the local reference to produce beat signal. Then the beat signal is fed into a high-pass filter to produce amplitude variation of beat1 signal by amplitude-frequency response. The envelope of beat1 signal is extracted by the feedback module and compared with a standard envelope of beat 2 signal to get the deviation of frequency (MATLAB program of envelope extraction runs in the background). After integration and amplification, the deviation signal $U$ is finally fed back to the circuit of standard triangular drive current.

As shown in Figure 4, there are two ranging systems including a nonlinear system with the quadratic modulation coefficient and an ideal system without the quadratic modulation coefficient as a reference. Except for the linear modulation coefficient and the quadratic modulation coefficient, the parameters of the corresponding device modules in the two FMCW ranging systems are the same.

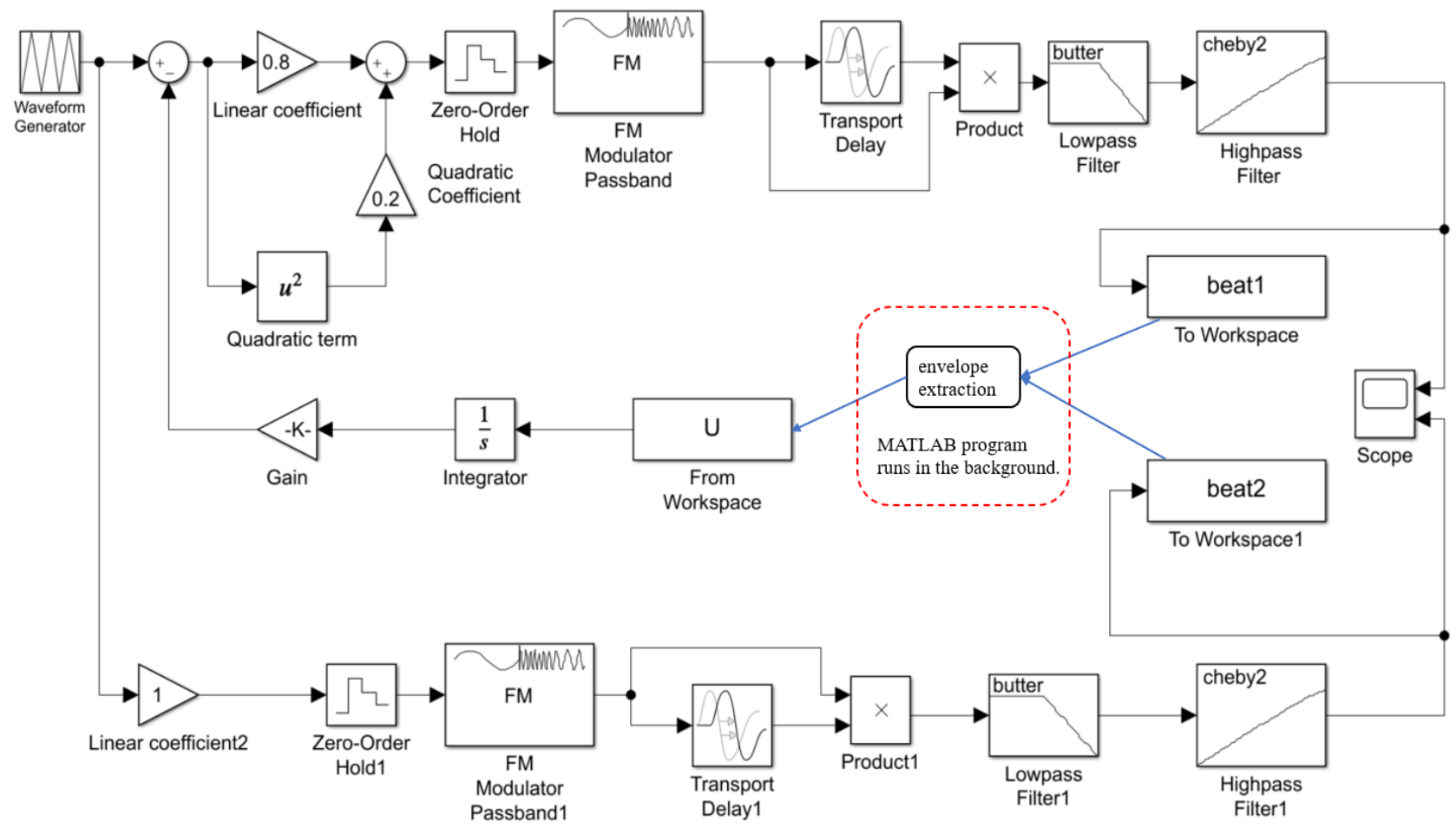

Figure 4. Simulation system of frequency sweep linearization using Simulink. 
Based on the parameters $a$ and $b$, the linear coefficient $a$ is equal to 0.8 and the quadratic coefficient $\mathrm{b}$ is equal to 0.2 , the nonlinear beat frequency is $4.576 \mathrm{MHz} \leq \Delta f_{b} \leq 6.864 \mathrm{MHz}$. For the ideal FMCW ranging, the linear modulation coefficient is equal to 1 and the quadratic modulation coefficient is equal to 0 , the beat frequency is $5.72 \mathrm{MHz}$, which is constant. The high-pass filter is selected based on the linearity of amplitude-frequency response and the range of $\Delta f_{b}, \Delta f_{b}$ should be close the center of the transition band, and its range relative to the transition band is small. The parameters of the designed high-pass filter are shown in Table 2.

\section{Simulation Results and Analysis}

The beat 1 and beat 2 signals correspond to nonlinear beat signal and ideal beat signal after the frequency-amplitude response by the high pass filter.

Figure 5 shows the time and frequency domain diagram of beat1 signal and beat 2 signal. It can be seen that the spectrum of beat 1 signal is broadened. And it can be easily compared that the amplitude of ideal beat 2 signal is invariable, while of beat 1 signal is similar to triangle change due to the frequency changes. The corresponding normalized envelopes are shown in Figure 6.
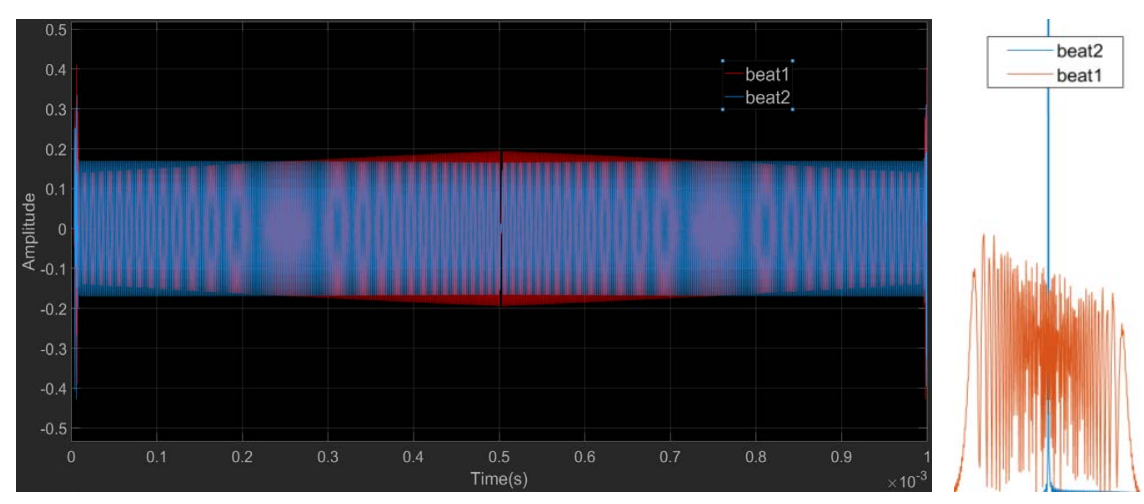

Figure 5. The time (left) and frequency (right) domain diagram of beat1 signal (red line) and beat2 signal (blue line).

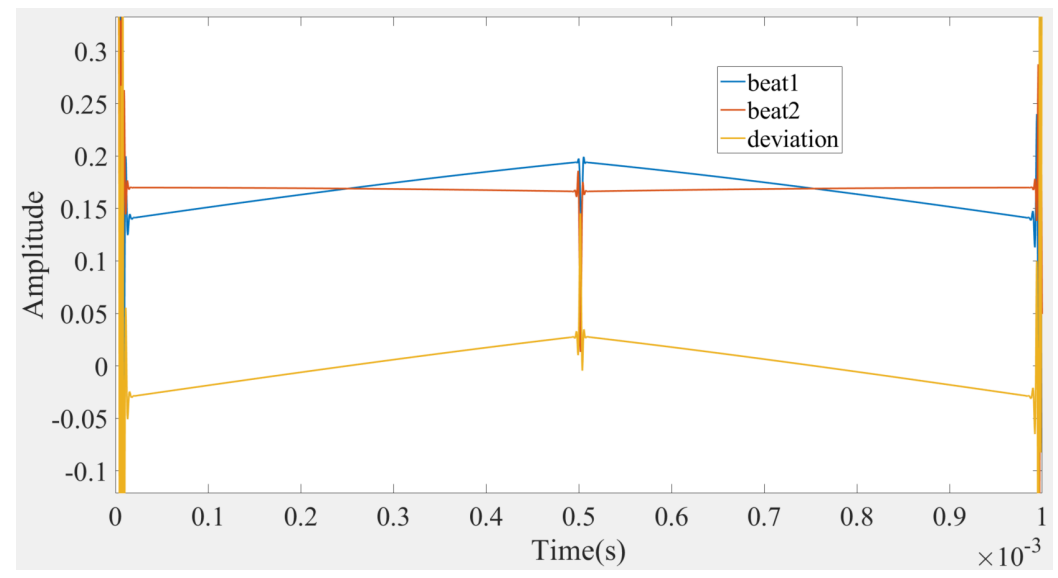

Figure 6. Schematic diagram of envelope deviation (yellow line) and envelope signals of beat2 signal (red line) and beat1 signal (blue line). 
Figure 6 shows the envelopes of the two signals (beat 1 and beat2), and the envelope deviation. We can find that the envelope is distorted at the inflection point of frequency sweep. In order to avoid the influence of the inflection point of frequency sweep, a small segment of envelope at the inflection point can be considered as ideal due to the small delay time, and the deviation signal at the inflection point can be expressed as 0 , as shown by the blue line in Figure 7.

In Figure 7, the signal (red line) is obtained by integrating the deviation signal (blue line), the feedback signal (yellow line) is 10,000 times the signal (red line).

Figure 8 shows the time domain diagram of beat1 signal (red line) and beat 1 signal with feedback gain (blue line). It can be easily compared that the amplitude of beat 1 signal with feedback gain tends to be stable and similar to that of the beat 2 signal due to the frequency sweep linearization.

The spectrum distributions of the nonlinear beat signals with different feedback gains are also investigated, as shown in Figure 9. The spectrum distributions of the nonlinear beat signals without linearization are depicted in red lines. The blue lines represent the spectrum distributions of beat signals with ideal and different feedback gains. Comparing these five spectrum distributions, the frequency sweep linearization method can make the spectrum distribution more concentrated and tend to the ideal state.

The nonlinear frequency modulation curve after frequency sweep linearization with gain of 10,000 is shown by the yellow line in Figure 10. The red line in Figure 10 represents the nonlinear frequency modulation curve without linearization and the blue line represents the ideal frequency modulation curve which is a standard triangle. The linearity (nonlinear error $\delta$ ) is obtained by the maximum deviation $\Delta f_{\max }$ between a nonlinear frequency modulation curve and the ideal frequency modulation curve. It can be expressed as

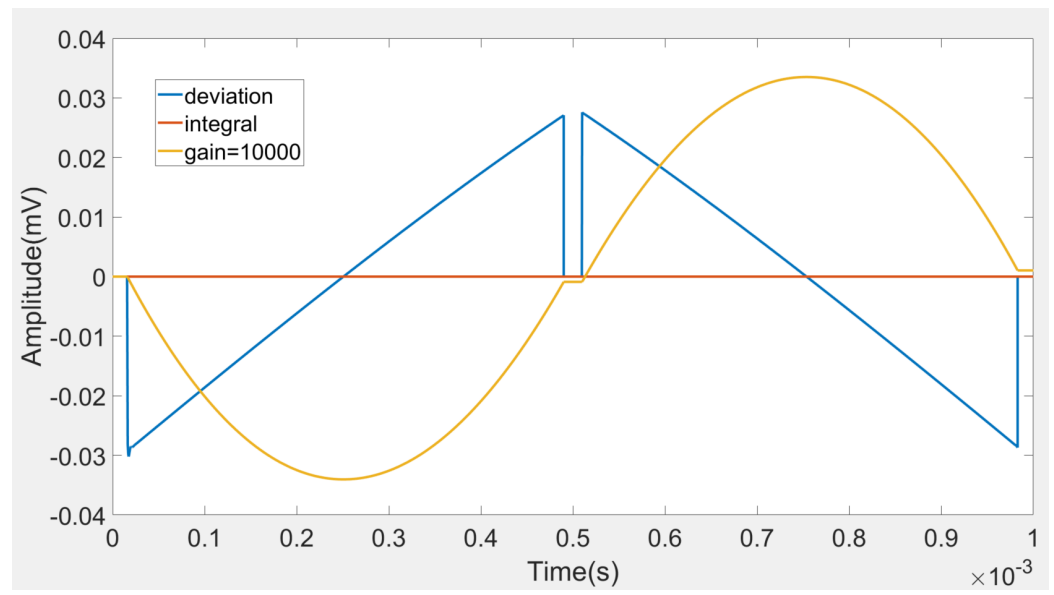

Figure 7. Schematic diagram of deviation signal processing.

Table 2. Parameters of the high-pass filter.

\begin{tabular}{ccccc}
\hline Parameters & Design method & Order & Stopband edge frequency & Stopband attenuation \\
\hline values & Chebyshev II & 1 & $1.6 \mathrm{MHz}$ & $20 \mathrm{~dB}$ \\
\hline
\end{tabular}




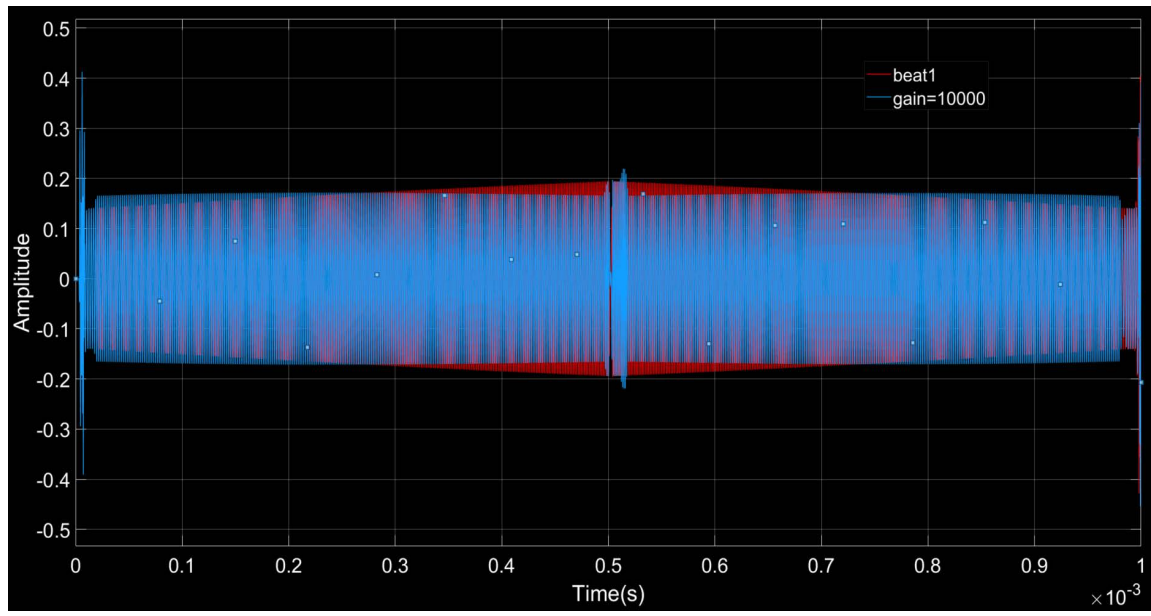

Figure 8. The time domain diagram of beat 1 signal and beat 1 signal with feedback gain.

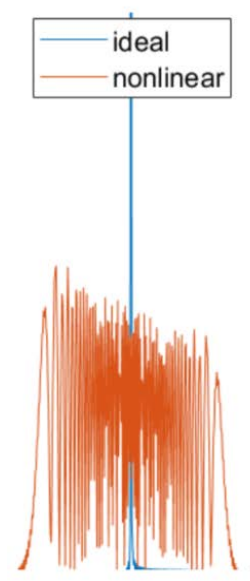

(a)

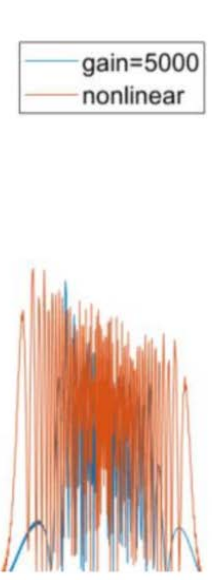

(b)

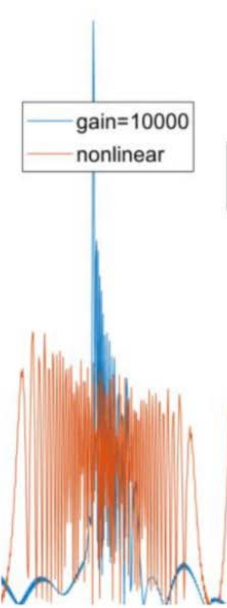

(c)

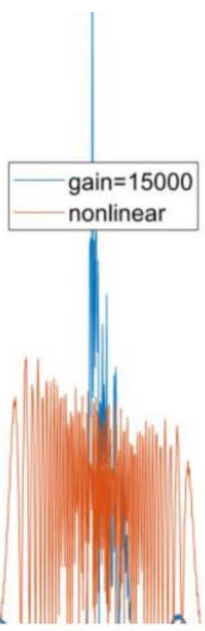

(d)

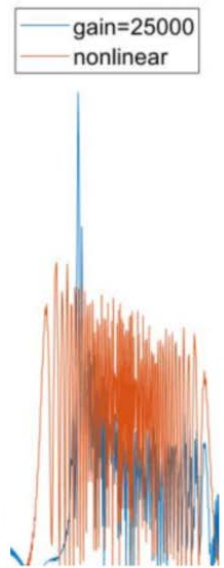

(e)

Figure 9. the spectrum distributions of beat signals with different feedback gains ((a) ideal; (b) 5000; (c) 10,000; (d) 15,000; (e) 25,000).

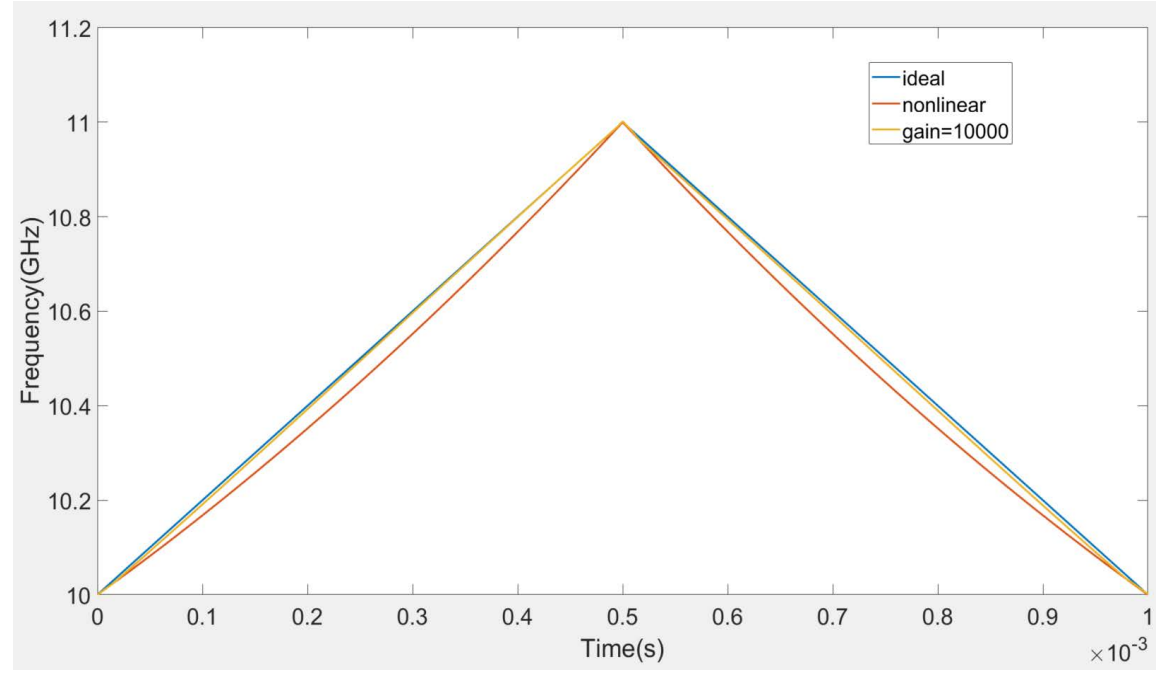

Figure 10. Comparison of three frequency modulation curves. 


$$
\delta=\Delta f_{\max } / \mathrm{B} \times 100 \%,
$$

where $\mathrm{B}=1 \mathrm{GHz}$. Then the linearity of the nonlinear frequency modulation curve without linearization is around $5.00 \%$. For the first feedback, the linearity with gain of 10,000 is around $1.69 \%$, the linearity with gain of 15,000 is $0.60 \%$. If iterative feedback is considered, Linearity will be better. The linearity with gain of 20,000 is $-2.07 \%$, this means that the gain is too large and the feedback signal is too strong.

\section{Conclusion}

A frequency sweep linearization scheme without post-processing processes is proposed in this paper. This scheme uses the frequency-amplitude response of high-pass filter and does not need expensive linear laser, complex circuit of photoelectronic PLL or reprocessing processes, thus can reduce the complexity of feedback system. Simulation results show that the linearity of nonlinear frequency modulation curve after frequency sweep linearization can reach around $0.60 \%$.

\section{Conflicts of Interest}

The authors declare no conflicts of interest regarding the publication of this paper.

\section{References}

[1] Behroozpour, B., Sandborn, P.A.M., Wu, M.C. and Boser, B.E. (2017) Lidar System Architectures and Circuits. IEEE Communications Magazine, 55, 135-142. https://doi.org/10.1109/MCOM.2017.1700030

[2] Roos, P.A., Reibel, R.R., Berg, T., Kaylor, B., Barber, Z.W. and Babbitt, Wm.R. (2009) Ultrabroadband Optical Chirp Linearization for Precision Metrology Applications. Optics letters, 34, 3692-3694. https://doi.org/10.1364/OFC.2010.OThQ2

[3] Iiyama, K., Wang, L.T. and Hayashi, K.I. (1996) Linearizing Optical Frequency-Sweep of a Laser Diode for FMCW Reflectometry. Lightwave Technology, 14, 173-178. https://doi.org/10.1109/50.482260

[4] Greiner, C., Boggs, B., Wang, T. and Mossberg, T.W. (1998) Laser Frequency Stabilization by Means of Optical Self-Heterodyne Beat-Frequency Control. Optics Letters, 23, 1280. https://doi.org/10.1364/OL.23.001280

[5] Satyan, N., Vasilyev, A., Rakuljic, G., Leyva, V. and Yariv, A. (2009) Precise Control of Broadband Frequency Chirps Using Optoelectronic Feedback. Optics Express, 17, 15991-15999. https://doi.org/10.1364/OE.17.015991

[6] Wu, Y., Chen, D. and Sun Y. (2013) Research on Optical Chirp Linearization Technique of Semiconductor Lasers by an Optoelectronic Feedback Loop. Chinese Journal of Lasers, 40, 1-6. https://doi.org/10.3788/CJL201340.0902001

[7] Zhang, X., Pouls, J. and Wu, M.C. (2019) Laser Frequency Sweep Linearization by Iterative Learning Pre-Distortion for FMCW LiDAR. Optics Express, 27, 9965-9974. https://doi.org/10.3788/CJL201340.0902001

[8] Zhang, T., Qu, X. and Zhang, F. (2018) Nonlinear Error Correction for FMCW Ladar by the Amplitude Modulation Method. Optics Express, 26, 11519. 
https://doi.org/10.1364/OE.26.011519

[9] Karlsson, C.J. and Olsson, F.A.A. (1999) Linearization of the Frequency Sweep of a Frequency-Modulated Continuous-Wave Semiconductor Laser Radar and the Resulting Ranging Performance. Applied Optics, 38, 3376-3386.

https://doi.org/10.1364/AO.38.003376

[10] Wang, H., Chen, J. and Chang, T. (2017) Research of Modulation Characteristics of Distributed Feedback Laser. Laser Technology, 41, 836-840. 